\title{
Removal time of postoperative vesical catheter in utero-vaginal prolapse surgery: a comparative study
}

\author{
(1) EDGARDO CASTILLO-PINO, (1) NATALIA BENAVIDES, (1) VALENTINA ACEVEDO, (1) VALERIA ALONSO
}

Pelvic Floor Unit, Department of Obstetrics and Gynaecology, University of the Republic School of Medicine, Clinical Hospital, Montevideo, Uruguay

\section{ABSTRACT}

Objective: To assess the efficacy and complications of early, intermediate, or late removal of the urinary catheter after vaginal hysterectomy, pelvic floor repair and anterior colporrhaphy.

Materials and Methods: Seventy-three Women with primary uterine or vaginal prolapse $\geq$ stage II according to Pelvic Organ Prolapse Quantifications System without stress urinary incontinence and without recurrent urinary tract infections, candidates for vaginal repair surgery were included. A urinary catheter (Foley 16) was inserted at the time of the intervention and it was removed at random in three groups, 24 (27 patients), 48 (23 patients) and 72 hours (23 patients) after surgery. Prophylactic intravenous antibiotics were administered for 72 hours. Urinary tract infection (UTI) was diagnosed by urine culture after surgery and acute urinary retention (AUR) during hospitalization. Percentage frequencies were calculated, and the chi-square test $\left(\mathrm{x}^{2}\right)$ was used to search for differences.

Results: UTI was observed in 7.4\%, 17.4\% and 13\% in groups 1, 2 and 3 respectively. No statistically significant association was found between the presence of UTI and urinary catheterization time $\left(x^{2}=1.3 p=0.512\right)$. AUR was found in $4.1 \%$ of all patients, most of them from group 2 .

Conclusion: Early removal of the urinary catheter in the first 24 hours after vaginal surgery decreased catheterization time, hospital stay and urinary tract infection. Extended catheterization does not offer benefits to patients and prolongs hospital stay unnecessarily.

Keywords: Urinary catheter; prolapse; vaginal surgery; urinary tract infection; post-operative urinary retention; urine culture

\section{INTRODUCTION}

Vaginal reconstruction surgery in pelvic organ prolapse (POP) has its own intra-operative complications, such as bleeding, vaginal infection, bladder, ureteral, or intestinal injury; and postoperative complications, such as febrile morbidity, urinary retention, and urinary tract infections. ${ }^{1,2}$ In vaginal prolapse surgery, transurethral bladder catheterization is used to control urinary output, reduce the possibility of bladder injury, and prevent post-operative urinary retention.
Traditionally, two types of urinary catheters are used. Transurethral catheters, that would remain in place for at least 24 hours to avoid acute post-operative urinary retention (AUR), and suprapubic catheters, placed through the abdomen to reduce the risk of urinary tract infection (UTI).

The duration of the catheter stay in the bladder has been reduced over the years. Generally, it is based on personalized knowledge rather than evidence-based knowledge. All this leads to the fact that the catheter durations in the bladder vary considerably. ${ }^{3}$ It

Address for Correspondence: Edgardo Castillo Pino, Pelvic Floor Unit, Department of Obstetrics and Gynecology, University of the Republic School of Medicine, Clinical Hospital, Montevideo, Uruguay

E-mail: castillopino@gmail.com ORCID: orcid.org/0000-0003-0241-9625

Received: 08 April 2021 Accepted: 16 April 2021 
is common to use routine bladder catheterization for up to three days after vaginal hysterectomy.

Prolonged catheterization increases the possibility of UTI, avoids early ambulation, prolongs hospital stay, and also has negative effects on postoperative well-being. ${ }^{3-7}$ In contrast, short-term catheterization reduces hospital stay, costs, and allows early mobilization after the operation..$^{8-10}$ The duration of bladder drainage to avoid urinary retention after gynecological surgery varies considerably. ${ }^{11}$ Early catheter removal can lead to AUR due to reflex pain at the operation site and overfilling of the bladder after prolapse surgery could have a negative effect on the surgical outcome. ${ }^{6}$ The duration of the catheter stay after the operation is based on custom rather than evidence. A Cochrane review of catheter policies after urogenital surgery was unable to make any consistent recommendations. ${ }^{12}$ Therefore, the objective of the present study was to evaluate the effectiveness and compare the postoperative complications of early, intermediate or late removal of the urinary catheter after vaginal cystocele repair surgery (with or without vaginal hysterectomy) and to determine the prevalence of asymptomatic UTI and AUR.

\section{MATERIALS AND METHODS}

A prospective, randomized study was designed. Women assisted by the medical team of the Pelvic Floor Unit were selected during the period from March 1 15t 2019 to March 15t 2020 . The study was approved by the Ethics Committee from the University of the Republic School of Medicine, Clinical Hospital from Montevideo, Uruguay (no: 81, date: November 29 ${ }^{\text {th }}$, 2017). All included patients signed a consent, after being informed about the study. The inclusion criteria were uterine or vaginal prolapse with asymptomatic primary cystocele $\geq$ stage II according to POP-Q with indication for site-specific vaginal repair surgery such as isolated anterior colporrhaphy or associated with vaginal hysterectomy or with Fothergill-Manchester and Richter procedures. Asymptomatic cystocele is the term referred to if there was no urinary incontinence.

Women with stage I prolapse, stress urinary incontinence, history of previous urinary retention, preoperative urinary tract infection, renal function compromise parameters (blood urea $>40 \mathrm{mg} / \mathrm{dl}$, serum creatinine $>1 \mathrm{mg} / \mathrm{dl}$ ), diabetics, those withintra-operative bladder injury and patients who did not give their consent, were excluded from the study.

The included patients were admitted to the hospital where a medical history and detailed physical examination were documented. Data on age, menopausal status, stage of prolapse (POP-Q) and type of intervention performed were recorded.
At the time of the intervention, a urinary catheter (Foley 16) was inserted in all patients. $71 \%$ of the patients underwent spinal anesthesia. After the intervention, a liquid diet was started followed by a normal diet. All patients received intravenous antibiotics for 3 days.

This randomized clinical trial included three groups of patients who underwent vaginal repair surgery with native tissues. All the patients were operated on by the same surgical team.

Randomization was performed with sequentially numbered, sealed envelopes prepared by an independent investigator.

After surgery, the urinary catheter was removed according to randomization in three groups, 24, 48, and 72 hours postoperatively, that is, groups 1,2 , and 3, respectively. After catheter removal, if the patient failed to spontaneously void, a re-catheterization was performed.

On the third day after the operation, a microscopic examination of urine and urine culture was systematically performed. Postoperative bacteriuria was defined as a positive urine culture of $>100,000 \mathrm{CFU} / \mathrm{ml}$.

Length of stay was defined as the time interval between surgery and discharge from hospital.

The variables analyzed were the re-catheterization rate to assess the risk of AUR during hospitalization, the risk of asymptomatic UTI by performing urine culture and the duration of hospital stay.

From the statistical analysis, the calculation of the percentage frequencies was considered, and the chi-square test $\left(x^{2}\right)$ was used to search for association.

\section{RESULTS}

In total, 73 women were recruited for this study. They were assigned to three groups with the following distribution, 27 in group 1, 23 in group 2 and 23 in group 3.

All patients had similar indications for vaginal surgery. The age and menopausal status are shown in Table 1.

There were no major intraoperative complications requiring a patient to be removed from the study protocol.Patients were divided into four age groups: less than 51 years, 51 to 60 years, 61 to 70 years and over 71 years old. The mean age was 66.55 (standard deviation \pm 10.97 ). Forty-seven patients $(64.4 \%)$ underwent vaginal hysterectomy with pelvic floor repair, 22 surgeries (30.1\%) with anterior colporrhaphy, two surgeries for repair of apical prolapse by Richter's operation (2.7\%) and two Manchester-Fothergill operations (2.7\%).

Most vaginal hysterectomies with pelvic floor repair were performed in the group of women 60 to 69 years of age. 95.9\% of cases are postmenopausal women. 
Preoperatively, the degree of prolapse according to the POP-Q quantification system was $20.5 \%$ with POP-Q II, $60.3 \%$ with POP-Q III and 19.2\% with POP-Q IV.

Postoperatively, UTI was observed in $7.4 \%, 17.4 \%$, and $13 \%$ in groups 1, 2, and 3, respectively (Figure 1). No statistically significant association was found between the presence of UTI and bladder catheterization time $\left(x^{2}=1.3 . p=0.512\right)$. It was observed that $4.1 \%$ of total patients presented with AUR, the majority was from group 2 (Table 2 )

Of twenty-seven patients in group 1,3.7\% had AUR and required re-catheterization (Figure 2). Of group 2, 8.3\% required recatheterization due to retention. No retention was observed in any of the patients in group 3 (Table 1).

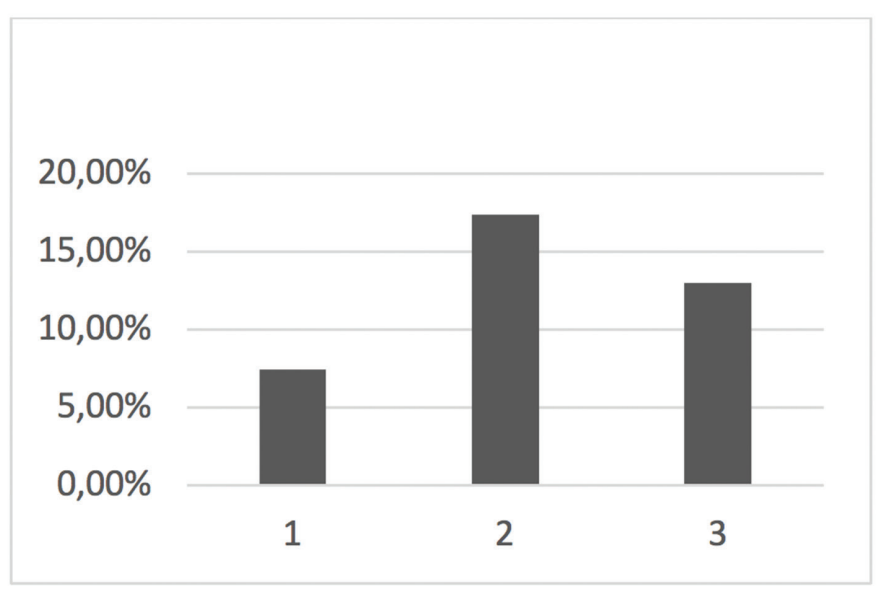

Figure 1 . UTI by assigned groups UTI: Urinary tract infection

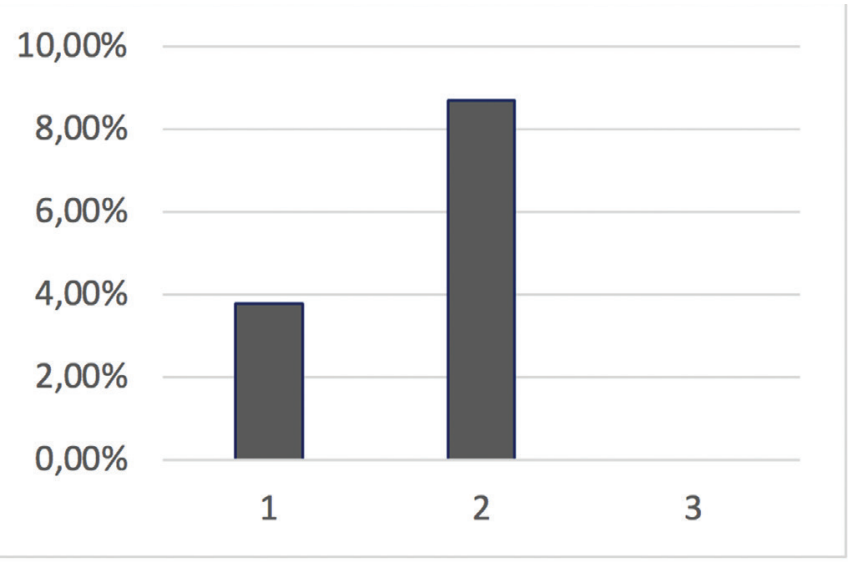

Figure 2. AUR by assigned groups

AUR: Acute urinary retention

There were no differences between the groups as the difference between UTI was not statistically significant. The hospital stay was three days for most patients, except for one patient in group 2 who had a five-day stay due to urinary retention.
Positive bacterial culture was found in $7.4 \%$ of patients in group 1, $17.4 \%$ in group 2, and 13\% in group 3 (Table 2). The most common bacteria was E. coli (67\%), followed by Klebsiella pneumoniae (22\%) and Proteus mirabilis (11\%).

\begin{tabular}{|l|l|l|l|l|}
\hline Table 1. Participant characteristics \\
\hline Groups & $\begin{array}{l}\mathbf{1}(\mathbf{2 4} \mathbf{H s}) \\
(\mathbf{n = 2 7 )}\end{array}$ & $\begin{array}{l}\mathbf{2}(\mathbf{4 8} \mathbf{H s}) \\
\text { (n=23) }\end{array}$ & $\begin{array}{l}\mathbf{3} \text { (72 Hs) } \\
\text { (n=23) }\end{array}$ & $\begin{array}{l}\text { Total } \\
\text { (n=73) }\end{array}$ \\
\hline Premenopausal & $11.10 \%$ & 0 & 0 & $4.10 \%$ \\
\hline Postmenopausal & $88.90 \%$ & $100 \%$ & $100 \%$ & $95.90 \%$ \\
\hline Age $\leq 50$ & $11.10 \%$ & 0 & 0 & $4.10 \%$ \\
\hline Age 51-60 & $14.80 \%$ & $21.70 \%$ & $13.00 \%$ & $16.40 \%$ \\
\hline Age 61-70 & $51.80 \%$ & $26 \%$ & $47.80 \%$ & $42.50 \%$ \\
\hline Age $\geq 71$ & $22.20 \%$ & $52 \%$ & $39.10 \%$ & $37 \%$ \\
\hline POP Q II & $23 \%$ & $16.70 \%$ & $21.70 \%$ & $20.50 \%$ \\
\hline POP Q III & $61.50 \%$ & $66.70 \%$ & $52.20 \%$ & $60.30 \%$ \\
\hline POP Q IV & $15.50 \%$ & $16.70 \%$ & $26.10 \%$ & $19.20 \%$ \\
\hline Regional anesthesia & $26 \%$ & $23 \%$ & $22 \%$ & $71 \%$ \\
\hline General anesthesia & $11 \%$ & $8 \%$ & $10 \%$ & $29 \%$ \\
\hline $\begin{array}{l}\text { Values are given as number (percentage) unless otherwise indicated } \\
\text { POP-Q: Pelvic Organ Prolapse Quantifications System; Hs: Hours; }:\end{array}$ \\
Number
\end{tabular}

Table 2. A comparison of the rates of UTI, AUR and hospital stay in the different groups

\begin{tabular}{|c|c|c|c|c|}
\hline Groups & $\begin{array}{l}1(24 \mathrm{Hs}) \\
(\mathrm{n}=27)\end{array}$ & $\begin{array}{l}2 \text { (48 Hs) } \\
(n=23)\end{array}$ & $\begin{array}{l}3 \text { (72 Hs) } \\
(n=23)\end{array}$ & $\begin{array}{l}\text { Total } \\
(n=73)\end{array}$ \\
\hline UTI & $7.4 \%$ & $17.4 \%$ & $13.00 \%$ & $12.3 \% *$ \\
\hline AUR & $3.8 \%$ & $8.3 \%$ & $0 \%$ & $4.1 \%$ \\
\hline $\begin{array}{l}\text { Hospital stay } \\
3 \text { days }\end{array}$ & $100 \%$ & $95.8 \%$ & $100 \%$ & $98.6 \%$ \\
\hline \multicolumn{5}{|l|}{$p=0.512$} \\
\hline \multicolumn{5}{|c|}{$\begin{array}{l}\text { *: The percentage frequencies and the value of p were calculated } \\
\text { UTI: Urinary tract infection; AUR: Acute urinary retention; Hs: Hour; } \\
\text { n: Number }\end{array}$} \\
\hline
\end{tabular}

\section{DISCUSSION}

Maintaining a urinary catheter in the bladder for an extended period of time during the postoperative period has changed over the years. Evidence and experience have shown that prolonging the duration of a urinary catheter had no additional benefit.

The urinary catheter is commonly used to assess urinary output and to prevent postoperative urinary retention. Bladder catheterization is not a harmless procedure. Hospital-acquired UTI is associated with the use of urinary catheters. UTI increases hospital stay, is expensive to treat, and causes discomfort to patients. 
A previous study compared catheter removal after one day and three days ${ }^{13}$ the authors of that study discussed perioperative considerations to prevent acute urine retention. Another study reported a group of patients who had their urinary catheters removed immediately after surgery. ${ }^{10}$ The authors recommended removing urinary catheter after three hours with careful monitoring of the patient's voiding.

The immediate removal of the catheter may cause difficulty in early ambulation and the recovery of bladder function due to the residual effect of regional anesthesia after using intra-spinal opioids.

In our study, these aspects were considered, and it was decided to remove the catheter 24 hours after the operation.

There are studies that show that the retention rate was higher in the prolonged catheterization group. . $^{14,15}$ However, other studies showed that retention rates were more common in the early removal group compared to the late removal group., $6,10,13,14,16-19$ Hakvoort et al. ${ }^{6}$ reported that in women with anterior colporrhaphy, if the catheter was removed within 24 hours, $40 \%$ required a new catheterization. Similarly, Alessandri et al. ${ }^{8}$, in a randomized clinical study in patients undergoing vaginal hysterectomy, found a relatively high rate of re-catheterization (18.8\%) in the immediate removal group.

In our study, re-catheterization due to urine retention was found in $4.1 \%$, being higher in group 2 (8.7\%).

Most studies report a higher incidence of UTI when the urinary catheter remains for longer. ${ }^{6,10,16} \mathrm{~A}$ reduction in UTIs is important since they represent $40 \%$ of all hospital-acquired infections and $80 \%$ of these are associated with the use of urinary catheters. ${ }^{19}$ Our study found a high incidence of UTI in all groups (7.4\%, 17.4\% and $13 \%$ ), but there were no statistically significant differences.

In 2004, a randomized clinical trial determined the benefits of prolonged urinary catheterization after prolapse surgery. ${ }^{6}$ One hundred patients were assigned into two groups. One group $(n=50)$ where the catheter was removed on the fifth day after the operation and the other $(n=50)$ where the removal was on the day after the operation. Positive urine cultures were found in $40 \%$ of cases in the prolonged catheterization group compared to $4 \%$ in the non-prolonged group (odds ratio: $15,95 \%$ confidence interval: 3.2-68.6). The authors concluded that removal of the urinary catheter is preferable in the morning after surgery and prolonged catheterization should only be performed when there are specific indications.

A systematic Cochrane review in 2006 of urinary catheter management after urogenital surgery in adults showed a lower rate of UTI when the catheter was removed early. ${ }^{12}$ The authors concluded that the use or not of a particular policy is generally based on the balance between the risks of morbidity (especially infection) and the risks of repositioning a catheter. ${ }^{12}$

Bacteriuria from a single bladder catheterization was observed in $3 \%$ to $4 \%$, while if catheterization was not required, there was a $1 \%$ risk of acquiring bacteriuria during hospitalization. ${ }^{20}$ Permanent catheters are particularly vulnerable to colonization by bacterial biofilms. ${ }^{21,22}$ Receiving prophylactic antibiotics reduces the rate of bacteriuria and other signs of infection in surgical patients who undergo bladder drainage for at least 24 hours after surgery. ${ }^{19}$ In 2010 , a study compared intermittent and suprapubic catheterization after anterior or posterior colporrhaphy. ${ }^{21}$ The length of hospital stay and total length of catheterization were significantly shorter for the intermittent group. ${ }^{21}$ In 2011, a randomized clinical trial assessed the management of abnormal residual volume after prolapse surgery, comparing the use of intermittent and transurethral catheterization. A twenty-fold reduction in the risk of urinary infection with intermittent catheterization was demonstrated. ${ }^{23}$ Furthermore, it was found that patients preferred intermittent catheterization. ${ }^{23}$

In 2017, a randomized controlled trial, ${ }^{24}$ compared immediate removal of the urinary catheter versus a suprapubic catheter after vaginal prolapse surgery. It was observed that a permanent catheter is not necessary in the postoperative period for many women.

Reduction in length of stay and early mobilization are consequent to avoiding a permanent catheter. ${ }^{25,8}$

The present randomized controlled trial was performed to compare immediate 24-hour removal versus use of a permanent catheter with removal at 48 and 72 hours.

The strengths of the study are being a prospective randomized study with defined inclusion and exclusion criteria, and that all the patients were operated on by the same surgical team. In addition, a study protocol with a similar length of hospital stay was followed in each group to reduce the chances of bias. The limitations were that various types of surgery were included, the sample size and the use of general anesthesia in some cases. Another limitation of the study was that groups 2 and 3 did not include premenopausal patients.

Our research has shown that it is not necessary to use a permanent urinary catheter for longer than 24 hours after vaginal surgery for pelvic organ prolapse. A short duration of catheterization with removal at 24 hours is safe and does not cause an increase in retention or urinary infection. Prolonged catheterization does not offer benefits to patients and unnecessarily lengthens hospital stay. 


\section{CONCLUSION}

Removal of the urinary catheter after 24 hours following vaginal hysterectomy with anterior colporrhaphy decreased hospital stay and UTI rates.

\section{Contributions}

Surgical and Medical Practices: E.C.P., N.B., Va.A., V.A., Concept: E.C.P., Design: E.C.P., N.B., Data Collection or Processing: E.C.P., N.B., Va.A., V.A., Analysis or Interpretation: E.C.P., N.B., Literature Search: V.A., Writing: E.C.P.

\section{Ethics}

Ethics Committee Approval: Ethics Committee from the University of the Republic School of Medicine, Clinical Hospital from Montevideo, Uruguay (no: 81, date: November 29 ${ }^{\text {th }}$, 2017) Informed Consent: All included patients signed a consent, after being informed about the study.

Peer-review: Externally peer-reviewed.

\section{DISCLOSURES}

Conflict of Interest: No conflict of interest was declared by the authors.

Financial Disclosure: The authors declared that this study received no financial support

\section{REFERENCES}

1. Hodges KR, Davis BR, Swaim LS. Prevention and management of hysterectomy complications. Clin Obstet Gynecol 2014; 57: 43-57.

2. Clarke-Pearson DL, Geller EJ. Complications of hysterectomy. Obstet Gynecol 2013; 121: 654-73.

3. Vierhout ME. Prolonged catheterization after vaginal prolapse surgery. Acta Obstet Gynecol Scand 1998; 77: 997-9.

4. Dunn TS, Shlay J, Forshner D. Are in-dwelling catheters necessary for 24 hours after hysterectomy? Am J Obstet Gynecol 2003; 189: 435-7.

5. Andersen JT, Heisterberg L, Hebjørn, et al. Suprapubic versus transurethral bladder drainage after colposuspension/vaginal repair. Acta Obstet Gynecol Scand 1985; 64: 139-43.

6. Hakvoort RA, Elberink R, Vollebregt A, Ploeg T, Emanuel MH. How long should urinary bladder catheterisation be continued after vaginal prolapse surgery? A randomised controlled trial comparing short term versus long term catheterisation after vaginal prolapse surgery. BJOG 2004;111:828-30.

7. Schiøtz HA, Tanbo TG. Postoperative voiding, bacteriuria and urinary tract infection with Foley catheterization after gynecological surgery. Acta Obstet Gynecol Scand 2006; 85: 476-81.

8. Alessandri F, Mistrangelo E, Lijoi D, Ferrero S, Ragni N. A prospective, randomized trial comparing immediate versus delayed catheter removal following hysterectomy. Acta Obstet Gynecol Scand 2006; 85: 716-20.

9. Dunn TS, Shlay J, Forshner D. Are in-dwelling catheters necessary for 24 hours after hysterectomy? Am J Obstet Gynecol 2003; 189: 435-7.

10. Glavind K, Mørup L, Madsen H, Glavind J. A prospective, randomised, controlled trial comparing 3 hour and 24 hour postoperative removal of bladder catheter and vaginal pack following vaginal prolapse surgery. Acta Obstet Gynecol Scand 2007; 86: 1122-5.

11. Schiøtz HA, Tanbo TG. Postoperative voiding, bacteriuria and urinary tract infection with Foley catheterization after gynecological surgery. Acta Obstet Gynecol Scand 2006; 85: 476-81.

12. Phipps S, Lim YN, McClinton S, Barry C, Rane A, N'Dow J. Short term urinary catheter policies following urogenital surgery in adults. Cochrane Database Syst Rev 2006; 2: CD004374.

13. Baldini G, Bagry H, Aprikian A, Carli F. Postoperative urinary retention: anesthetic and perioperative considerations. Anesthesiology 2009; 110: 1139-57.

14. Summitt RL Jr, Stovall TG, Bran DF. Prospective comparison of indwelling bladder catheter drainage versus no catheter after vaginal hysterectomy. Am J Obstet Gynecol 1994; 170: 1815-8.

15. Sekhavat L, Farajkhoda T, Davar R. The effect of early removal of indwelling urinary catheter on postoperative urinary complications in anterior colporrhaphy surgery. Aust N Z J Obstet Gynaecol 2008; 48: $348-52$

16. Kamilya G, Seal SL, Mukherji J, Bhattacharyya SK, Hazra A. A randomized controlled trial comparing short versus long-term catheterization after uncomplicated vaginal prolapse surgery. J Obstet Gynaecol Res 2010; 36: 154-8.

17. Dunn TS, Shlay J, Forshner D. Are in-dwelling catheters necessary for 24 hours after hysterectomy? Am J Obstet Gynecol 2003; 189: 435-7.

18. Liang CC, Lee CL, Chang TC, Chang YL, Wang CJ, Soong YK. Postoperative urinary outcomes in catheterized and noncatheterized patients undergoing laparoscopic-assisted vaginal hysterectomy--a randomized controlled trial. Int Urogynecol J Pelvic Floor Dysfunct 2009; 20: 295-300.

19. Lusardi G, Lipp A, Shaw C. Antibiotic prophylaxis for short-term catheter bladder drainage in adults. Cochrane Database Syst Rev 2013; 2013: CD005428.

20. Nicolle LE. Catheter-related urinary tract infection. Drugs Aging 2005; 22: 627-39.

21. Stickler DJ. Bacterial biofilms in patients with indwelling urinary catheters. Nat Clin Pract Urol 2008; 5: 598-608.

22. Schiøtz HA. Comparison of 1 and 3 days' transurethral Foley catheterization after vaginal plastic surgery. Int Urogynecol J 1995; 6: $158-61$.

23. Hakvoort RA, Thijs SD, Bouwmeester FW, et al. Comparing clean intermittent catheterisation and transurethral indwelling catheterisation for incomplete voiding after vaginal prolapse 
surgery: a multicentre randomised trial. BJOG 2011; 118: 105560.

24. Bray R, Cartwright R, Digesu A, Fernando R, Khullar V. A randomised controlled trial comparing immediate versus delayed catheter removal following vaginal prolapse surgery. Eur J Obstet Gynecol Reprod Biol 2017; 210: 314-8.

25. Dixon L, Dolan LM, Brown K, Hilton P. RCT of urethral versus suprapubic catheterization. Br J Nurs 2010; 19: 7-13. 